\title{
Phosphorylation of Highly Conserved Neurofilament Medium KSP Repeats Is Not Required for Myelin-Dependent Radial Axonal Growth
}

\author{
Michael L. Garcia, ${ }^{1,2}$ Mala V. Rao, ${ }^{6,7}$ Jiro Fujimoto, ${ }^{2}$ Virginia B. Garcia, ${ }^{1}$ Sameer B. Shah, ${ }^{8}$ John Crum, ${ }^{4}$ Takahiro Gotow, ${ }^{9}$ \\ Yasuo Uchiyama, ${ }^{10}$ Mark Ellisman, ${ }^{3,4}$ Nigel A. Calcutt, ${ }^{5}$ and Don W. Cleveland ${ }^{2,3}$ \\ ${ }^{1}$ Department of Biological Sciences, Bond Life Sciences Center, University of Missouri-Columbia, Columbia, Missouri 65211, ${ }^{2}$ Ludwig Institute for Cancer \\ Research, ${ }^{3}$ Department of Neurosciences, ${ }^{4}$ National Center for Microscopy and Image Research, and ${ }^{5}$ Department of Pathology, University of California, \\ San Diego, La Jolla, California 92093, ${ }^{6}$ Nathan Kline Institute, ${ }^{7}$ Department of Psychiatry, New York University School of Medicine, Orangeburg, New York \\ 10962, ${ }^{8}$ Fischell Department of Bioengineering, University of Maryland, College Park, Maryland 20742, ${ }^{9}$ Laboratory of Cell Biology, College of Nutrition, \\ Koshien University, Hyogo 665-0006, Japan, and ${ }^{10}$ Department of Cell Biology and Neuroscience, Juntendo University School of Medicine, Bunkyo-ku, \\ Tokyo 113-8421, Japan
}

Neurofilament medium (NF-M) is essential for the acquisition of normal axonal caliber in response to a myelin-dependent "outside-in" trigger for radial axonal growth. Removal of the tail domain and lysine-serine-proline (KSP) repeats of NF-M, but not neurofilament heavy, produced axons with impaired radial growth and reduced conduction velocities. These earlier findings supported myelindependent phosphorylation of NF-M KSP repeats as an essential component of axonal growth. As a direct test of whether phosphorylation of NF-M KSP repeats is the target for the myelin-derived signal, gene replacement has now been used to produce mice in which all serines of NF-M's KSP repeats have been replaced with phosphorylation-incompetent alanines. This substitution did not alter accumulation of the neurofilaments or their subunits. Axonal caliber and motor neuron conduction velocity of mice expressing KSP phosphoincompetent NF-M were also indistinguishable from wild-type mice. Thus, phosphorylation of NF-M KSP repeats is not an essential component for the acquisition of normal axonal caliber mediated by myelin-dependent outside-in signaling.

Key words: neurofilaments; cytoskeleton; myelination; radial growth; motor neuron; phosphorylation

\section{Introduction}

Myelination occurs around axons that are $>1 \mu \mathrm{m}$ (Duncan, 1934). This is important for signal transmission since myelination is more efficient at increasing nerve conduction velocity than diameter increase alone (Rushton, 1951). The relationship between axons and myelinating cells is a reciprocal one, with each cell type sending and receiving signals from the other cell (de Waegh et al., 1992; Stevens and Fields, 2000). One such signal originates from myelinating cells and results in a large increase in

\footnotetext{
Received Aug. 9, 2008; revised Dec. 22, 2008; accepted Dec. 29, 2008.

This work was supported by National Institutes of Health (NIH) Grants NS 27036 to D.W.C. and DK 057629 to N.A.C., and National Science Foundation Grant MCB-0544602 to M.L.G. Salary support for D.W.C. was provided by the Ludwig Institute for Cancer Research. Salary support for M.L.G. was provided by the University of MissouriColumbia and the Bond Life Sciences Center. Some of the imaging was conducted at the National Center for Microscopy and Imaging Research at San Diego, which is supported by the NIH through a National Center for Research Resources program grant (P41 RR04050) awarded to M. Ellisman. We thank Monica Burgers for assistance with tissue preparation for light microscopic morphometric analysis and Brian Reigle for writing the scripts used in automated determination of numbers of axons and numbers of neurofilaments in assigned groups for axonal calibers and nearest-neighbor distances. We also thank Steve Lamont for writing software used in automated determination of nearest-neighbor distances and Josh Hartley for computer support and for assistance with generating publication-quality figures.

Correspondence should be addressed to Dr. Don W. Cleveland, Ludwig Institute for Cancer Research, University of California, San Diego, 9500 Gilman Drive, La Jolla, CA 92903. E-mail: dcleveland@ucsd.edu.

DOI:10.1523/JNEUROSCI.3765-08.2009

Copyright $\odot 2009$ Society for Neuroscience $\quad$ 0270-6474/09/291277-08\$15.00/0
}

axonal diameter (de Waegh et al., 1992). Assembly of compact myelin is essential for initiation of radial axonal growth (de Waegh et al., 1992), yielding nearly stoichiometric phosphorylation of neurofilament KSP repeats on both neurofilament medium (NF-M) and neurofilament heavy (NF-H) subunits (Julien and Mushynski, 1982; de Waegh et al., 1992). Unmyelinated regions of the same axon retain smaller diameters, contain neurofilaments that are less phosphorylated, and more compact (Hsieh et al., 1994).

Neurofilaments are obligate heteropolymers of neurofilament light (NF-L), medium (NF-M), and heavy (NF-H) subunit proteins (Lee et al., 1993). Within axons, neurofilaments are involved in establishing and maintaining the three-dimensional array of axoplasm that is required for radial growth. Loss of all axonal neurofilaments through spontaneous mutation in quail (Ohara et al., 1993) or by targeted deletion in mouse (Zhu et al., 1997) resulted in smaller caliber myelinated axons with slowed conduction velocities (Sakaguchi et al., 1993; Kriz et al., 2000). Moreover, several lines of evidence have supported neurofilament phosphorylation as the key component of neurofilamentdependent radial growth (de Waegh et al., 1992; Yin et al., 1998). Replacement of NF-M (Garcia et al., 2003; Rao et al., 2003) or both NF-M and NF-H (Garcia et al., 2003) with versions missing the C-terminal tails that extend from the filament surface and 

ekeeepeaeKSPvKSPeakeeeeegekeeeeegqeeeeedegvKSDqaeeggsekegssekdegeqeeeegeteae gegeeaeakeekkiegkveevavkeeikvekpekaKSPmpKSPveevkpkpeakagkgeqkeeekveeekkevtKESPk eekvekkeekpkdvadkkKAESPvkekaveevitisksvkvslekdtkeekpqpqekvkekaeeeggseeegsdrspqeskke diaingevegkeeeeqetqekgsgreeekgvvtngldvspaeekkgedssddkvvvtkkvekitseggdgatkyitksvtvtqkvee heetfeeklvstkkvekvtshaivkevtqgd ${ }^{849}$
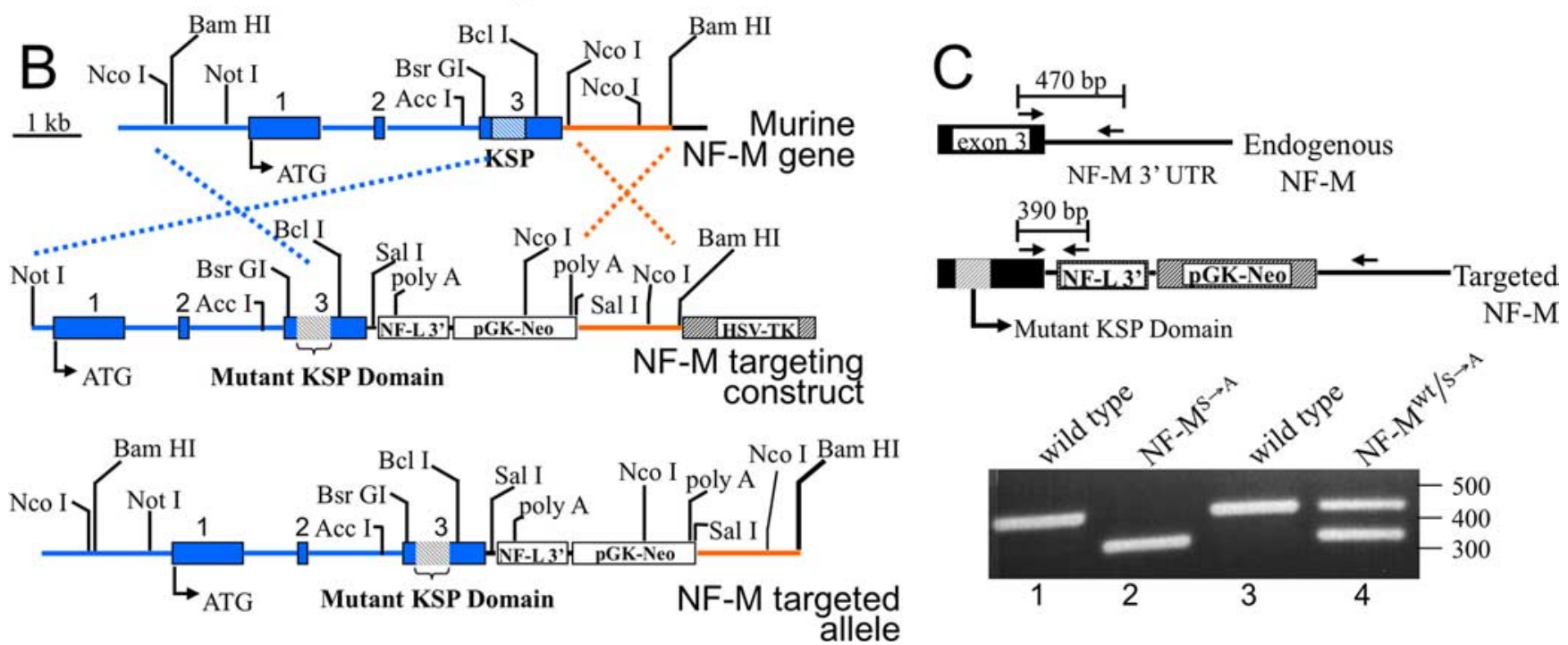

Figure 1. Generation of a KSP phospho-incompetent NF-M mouse by substituting all KSP serine residues with alanine (NF-M $\left.{ }^{S \rightarrow A}\right)$. $\boldsymbol{A}$, Mouse NF-M tail domain. The conserved sequence "KLLEGEE" (light blue) marks the end of the rod domain for all three neurofilament subunits. All KSP, KXSP, and KXXSP motifs are highlighted in bold, capital letters, as well as the variant KSD. The six previously identified highly phosphorylated KSP (Xu et al., 1992) motifs are indicated in red, and the nonphosphorylated KSP is indicated in dark blue. NF-M NCBI accession number NP_032717. $B$, Construction of an NF-M ${ }^{S \rightarrow A}$ allele in which the serine residues of the highly conserved KSP repeats were mutated to alanine residues. The three exons of the NF-M gene are indicated by the filled boxes interrupted by two introns. ATG denotes the translation initiation codon. Dotted lines indicate the two regions where homologous recombination could take place between the targeting vector and the endogenous NF-M allele. C, Mouse genomic DNA was screened for targeting of the serine to alanine NF-M mutant using three primers for PCR-based genotyping. Top, A schematic representation of the endogenous NF-M gene depicts the approximate location of primer annealing sites of two of the three PCR primers with the predicted size of the amplified region of NF-M. Middle, A schematic representation of the targeted NF-M gene depicts the approximate location of primer annealing sites for all of the primers. The predicted size for the smaller amplified region is indicated. Bottom, Genotyping the NF-M loci by PCR of genomic DNA isolated from mouse-tail biopsies.

contain all of the KSP repeat motifs resulted in axons with diminished radial axonal growth (Garcia et al., 2003; Rao et al., 2003). Surprisingly, loss of the more heavily phosphorylated NF-H tail domain alone did not affect normal axon caliber (Rao et al., 2002). Together these previous results demonstrated that the 426 aa NF-M tail plays an essential role in radial axonal growth and lead to the prediction that the target for the myelin-initiated outside-in signaling cascade is the six highly phosphorylated serine residues in this NF-M tail region that have been identified in cultured rat dorsal root ganglia (Xu et al., 1992). Of these identified sites, five are conserved KSP or KXXSP motifs and the other is a variant of this motif, KSD (Xu et al., 1992). We have now used gene replacement to substitute all KSP serines (including all KSP, KXSP, KXXSP) with alanine to generate mice that express KSP phosphorylation-incompetent NF-M. Analysis of these animals provides a direct test of the model in which myelination-dependent phosphorylation cascade targets NF-M KSP repeats as an essential feature of radial axonal growth.

\section{Materials and Methods}

Site-directed mutagenesis and construction of NF-M $M^{S \rightarrow A}$ mice by ES cellmediated knock-in. A genomic clone isolated from mouse 129 SVJ library was used for construction of NF-M ${ }^{\mathrm{S} \rightarrow \mathrm{A}}$ [provided by J. P. Julien, Laval University, Quebec, Quebec, Canada (Jacomy et al., 1999)]. A 7 kb NotIBamHI fragment of NF-M genomic clone was digested with AccI-BclI to produce $\mathrm{a} \sim 1.1 \mathrm{~kb}$ fragment that included all identified phosphorylation sites. Five oligonucleotides were designed to convert the phosphorylated serine residues to alanine using a PCR approach. The primers were phosphorylated and used for PCR-based mutagenesis using the QuikChange (Stratagene) mutagenesis reaction conditions. Following PCR, plasmids were transfected and mutations verified by DNA sequence analysis.

An AccI-BclI fragment that contained seven mutated sites was recloned into the NF-M gene to produce a full-length phosphoincompetent NF-M. Finally, the NF-L 3' untranslated region and a PGKNeo cassette for positive selection were cloned at the $3^{\prime}$ end (into the SalI site of the NF-M 3' untranslated region). Introduction of the NF-L/Neo cassette resulted in the generation of a $5 \mathrm{~kb}$ long arm and a $1.5 \mathrm{~kb}$ short arm to promote homologous recombination. At the end of the $3^{\prime}$ arm, an HSV-TK cassette was ligated to provide for negative selection of ES cell clones, thereby creating a final NF-M ${ }^{\mathrm{S} \rightarrow \mathrm{A}}$ targeting vector (Fig. $1 \mathrm{~B}$ ). After linearization with NotI, the DNA was electroporated into RI ES cells, provided by Andreas Nagy (University of Toronto, Toronto, Ontario, Canada), and selected with G418 and ganciclovir at $250 \mu \mathrm{g} / \mathrm{ml}$ and $2 \mu \mathrm{M}$, respectively (Joyner, 1994). Drug-resistant colonies were amplified and screened using PCR with a $5^{\prime}$ primer anchored in the Neo gene and a $3^{\prime}$ primer located outside the targeting construct yielding an $\sim 1.8 \mathrm{~kb}$ band upon successful recombination. Additionally, the DNA was digested with $\mathrm{NcoI}$ from PCR positive colonies, separated on $0.8 \%$ agarose gels, transferred to Hybond $\mathrm{N}$ filters (Amersham Biosciences), and hybridized with random prime-labeled $0.8 \mathrm{~kb}$ BamHI-NotI fragments. DNA sequence analysis was used to ensure incorporation of all seven mutant sites. One out of $150 \mathrm{ES}$ clones was identified to have undergone homologous recombination at both the $5^{\prime}$ and $3^{\prime}$ ends of the gene to produce NF-M ${ }^{\mathrm{S} \rightarrow \mathrm{A}}$ gene as indicated in Figure 1.

Detection and quantification of neurofilament and tubulin proteins by 
immunoblotting. Sciatic nerve extracts were made as previously described (Garcia et al., 2003). Protein concentration was determined using bicinchoninic acid assay kit (Pierce Chemical). Protein extracts were separated on 7\% polyacrylamide gels with SDS and transferred to nitrocellulose membranes (Lopata and Cleveland, 1987). The NF-H and NF-L subunits were identified using affinity-purified rabbit polyclonal antibodies $\mathrm{pAb}-\mathrm{NF}-\mathrm{HCOOH}$ and $\mathrm{pAb}-\mathrm{NF}-\mathrm{LCOOH}$ raised against the COOH-terminal 12 aa of mouse NF-H and NF-L, respectively (Xu et al., 1993). mAbs to NF-M [RMO44 (Tu et al., 1995) and neuron-specific, class III $\beta$-tubulin (T8660)] were used. Degree of phosphorylation of both NF-M and NF-H was determined using the monoclonal antibodies SMI-31 (Sternberger Monoclonals) and RT-97 (Rao et al., 2002). Secondary antibodies were goat anti-rabbit (for polyclonal primary antibodies, Sigma-Aldrich) or goat anti-mouse IgG (for monoclonal primary antibodies, Sigma-Aldrich) conjugated to HRP. Immunoreactive bands were visualized by ECL (Perkin-Elmer) followed by autoradiography.

Tissue preparation, morphological analysis, and nearest-neighbor analysis. Mice were perfused intracardially with $2.5 \%$ glutaraldehyde and $4 \%$ formaldehyde in $0.1 \mathrm{~m}$ Sorenson's phosphate buffer, $\mathrm{pH} 7.2$, and postfixed overnight in the same buffer. Samples were treated with $2 \%$ osmium tetroxide, washed, dehydrated, and embedded in Epon-Araldite resin as previously described (Garcia et al., 2003). Thick sections (0.75 $\mu \mathrm{m})$ for light microscopy were stained with p-phenylene diamine. Cross sections of L5 motor axons were analyzed in five to six mice per genotype and each age group. Axonal diameters were measured using the AxioVision Digital Image Processing Software (Carl Zeiss MicroImaging). Entire roots were imaged, imaging thresholds were selected individually, and the crosssectional area of each axon was calculated and reported as a diameter of a circle of equivalent area. Axon diameters were grouped into $0.5 \mu \mathrm{m}$ bins.

Thin sections (60-90 nm) were cut from prepared resin blocks with a Leica Ultracut E ultramicrotome, stained with $1 \%$ aqueous uranyl acetate for $15 \mathrm{~min}$. followed by lead salts for $2 \mathrm{~min}$. Images of selected neurons were collected at $80 \mathrm{kV}$ with a JEOL $1200 \mathrm{FX}$ at the indicated magnification of 5000, 10,000, or 20,000. Negatives were scanned at $1000 \mathrm{dpi}, 8 \mathrm{bit}$ depth with a Nikon CoolScan 9000 film scanner. Neurofilaments were traced and nearest-neighbor calculations made.

Means were analyzed for statistical significance using unpaired Student's $t$ test on total axon counts for wild-type versus NF-M ${ }^{\mathrm{S} \rightarrow \mathrm{A}}$ mice at 2 and 6 months. Bimodal distributions of motor axon diameter distributions were analyzed for overall statistical significance using Mann-Whitney $U$ test.

Visualization of neurofilament organization in the axon by quick-freeze, deep-etch analysis. Sciatic nerves of adult $\mathrm{NF}-\mathrm{M}^{\mathrm{S} \rightarrow \mathrm{A}}$ and their control littermate animals were dissected and incubated in oxygenated artificial CSF containing (in mM, pH 7.3) $126 \mathrm{NaCl}, 22 \mathrm{NaHCO}_{3}, 1 \mathrm{Na}_{2} \mathrm{HPO}_{4}, 2.8$ $\mathrm{KCl}, 0.88 \mathrm{MgCl}_{2}, 1.45 \mathrm{CaCl}_{2}$, and 3.5 glucose. Subsequently, nerves were sectioned with a razor blade, and the tissue was frozen by slamming against a liquid helium-cooled copper block (Hitachi HIF-4KOI) as previously reported (Gotow et al., 1999). The frozen tissue was mounted onto the freeze fracture apparatus (BAF 060, BAL-TEC), fractured, and then deep etched and rotary replicated with platinum/carbon at an angle of $25^{\circ}$. The replicas were examined with a Hitachi H-7100 electron microscope at $75 \mathrm{kV}$.

Nerve conduction velocity measurements. Nerve conduction velocities were measured in the sciatic nerve, interosseus muscle system of 6-month-old mice (Garcia et al., 2003). In brief, mice were anesthetized with Metophane (4\% in $\mathrm{O}_{2}$ for induction, 2-3\% for maintenance), and temperature was maintained at $37^{\circ} \mathrm{C}$ by a heating lamp and thermal pad connected to a temperature regulator and the rectal thermistor probe. The sciatic nerve was stimulated with single supramaximal square wave pulses (4-8 V and $0.05 \mathrm{~ms}$ duration; Grass Technologies) via fine needle electrodes placed at the sciatic notch and Achilles tendon. Evoked electromyograms were recorded from the interosseus muscles of the ipsilateral foot via two fine needle electrodes and displayed on a digital storage oscilloscope (Tektronix). The distance between the two sites of stimulation was measured using calipers, and conduction velocity was calculated as previously described (Calcutt et al., 1990). Measurements were made in triplicate from five animals per genotype, and the median was used as the measure of velocity. Statistical analysis was performed by unpaired $t$ test.
Methods for calculating neurofilament clustering. Average neurofilament spacing was determined by distributing identified neurofilaments in uniform arrays across the effective cross-sectional area of an axon. Cross-sectional area was estimated by tracing axoplasmic regions of the same digitized electron micrographs used to identify neurofilaments. Neurofilaments were organized in concentric hexagonal "rings" of equilateral triangles, with average neurofilament spacing calculated as the side length of one triangle. Hexagons were selected due to their inherent ability to pack two-dimensional space optimally (Conway and Sloane, $1999)$. The number of counted neurofilaments $\left(n_{\mathrm{NF}}\right)$ corresponded to $n_{\text {tri }}$ triangles, arranged into $i$ hexagonal rings. The relationships between $n_{\mathrm{NF}}, n_{\mathrm{tri}}$, and $i$ are given by the following recursive formulae: $n_{\mathrm{NF}}(i)=$ $n_{\mathrm{NF}}(i-1)+6 \times i$ and $n_{\mathrm{tri}}(i)=n_{\mathrm{tri}}(i-1)+6 \times[2 \times(i-1)+1]$, with $n_{\mathrm{NF}}(1)=7$ and $n_{\text {tri }}(1)=6$. Intermediate values (complete triangles within incomplete hexagonal rings) were calculated using interpolation. Average filament spacing was then calculated from the area of a single regular hexagon, which was determined by dividing the axonal crosssectional area by $n_{\text {tri }}$. Neurofilament clustering was defined as the ratio of average filament spacing to nearest-neighbor filament spacing, with higher ratios implying more clustered (less uniformly distributed) neurofilaments. Analysis was performed using MATLAB 6.5 (The MathWorks).

Sciatic nerve regeneration. Mice were placed under Metophane anesthesia, and the sciatic nerve was exposed via an incision in the flank followed by separation of underlying musculature by blunt dissection. The nerve was crushed using fine jewelers forceps at the level of the obturator tendon. To assess functional recovery of the injured limb, the mouse was induced to spread its toes by briefly lifting the hindlimbs off the bench. The distance from the first to fifth digits was measured with a divider and expressed as a percentage of preinjury spread distance.

Activity wheels. Mice were placed in a single activity wheel chamber system (Lafayette Instruments) for $14 \mathrm{~d}$. Activity was measured by the number of revolutions an animal would run during a $12 \mathrm{~h}$ period. Revolutions were counted using an optical sensor that detects wheel motion and were stored on an activity wheel counter (Lafayette Instruments). Revolutions were converted into kilometers based on a 5 -inch-diameter activity wheel. Statistical analysis was performed by unpaired $t$ test.

\section{Results \\ Site-directed mutagenesis and stoichiometric replacement of NF-M with phospho-incompetent NF-M}

For site-directed mutagenesis, a portion of the murine NF-M gene containing exon 3 (in a $\sim 1.1 \mathrm{~kb} A c c \mathrm{l} / B c \mathrm{l}$ fragment) was subcloned into pBluescript. Five primers were designed to alter the codons of the serine residue to alanine for all identified KSP, KXSP, and KXXSP motifs and the single KSD variant (Fig. 1A). DNA sequence analysis confirmed the substitution of the 7 serine codons with alanine codons without the introduction of additional sequence changes. The modified NF-M fragment was recloned back into a targeting vector that contained the $3^{\prime}$ half of the NF-M gene, including an added $3^{\prime}$ untranslated region and polyadenylation signal from the murine NF-L gene and a neomycin phosphotransferase gene for selection (Fig. $1 B$ ). After electroporation, one out of $150 \mathrm{ES}$ cell clones were identified to have correctly recombined the mutant NF-M allele into one of the endogenous NF-M alleles. This clone was injected into C57BL/6 blastocysts, producing chimeric animals that when bred to C57BL/6J mice transmitted the phospho-KSP-incompetent NF-M (referred to as NF-M ${ }^{S \rightarrow A}$ ) allele to their progeny. Mating pairs of mice heterozygous for the NF-M ${ }^{S \rightarrow A}$ allele produced homozygous animals (Fig. 1C) at the expected Mendelian frequency. The homozygous progeny were viable and fertile with no overt phenotype through 2 years of age, the oldest age analyzed.

Coomassie blue staining of sciatic nerve extracts indicated accumulation of phospho-KSP-incompetent NF-M in NF$\mathrm{M}^{\mathrm{S} \rightarrow \mathrm{A}}$ homozygous animals to levels indistinguishable from the 


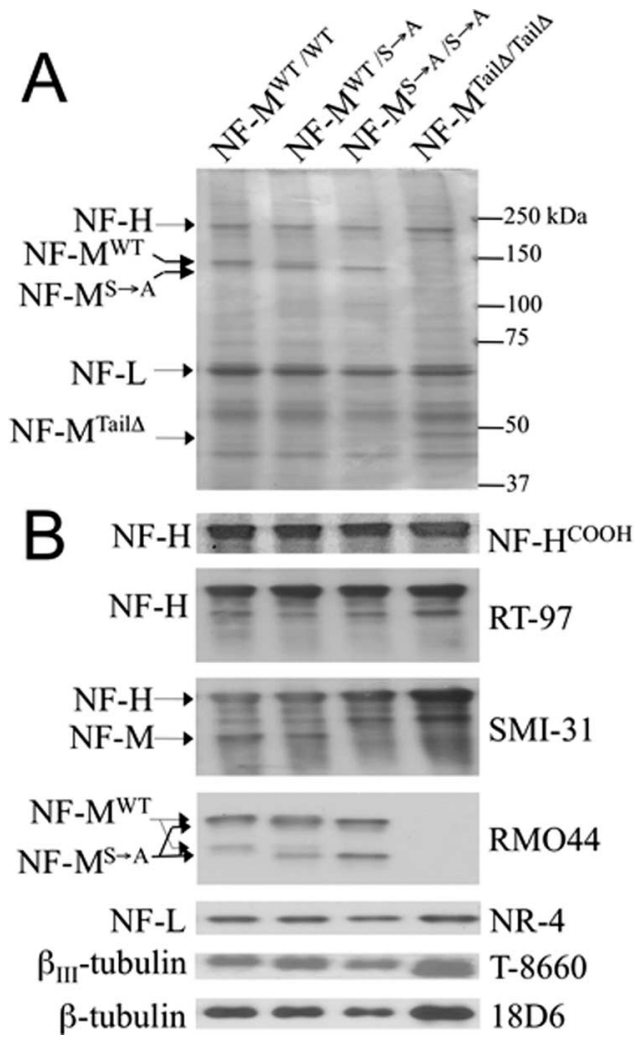

Figure 2. Substitution of NF-M ${ }^{S \rightarrow A}$ for wild-type NF-M does not affect stoichiometry of the neurofilament subunits. Expression of phospho-KSP-incompetent NF-M has no effect on relative stoichiometries and accumulated levels of NF-L, NF-M, NF-H, or tubulin. Parallel immunoblots of sciatic nerve extracts from 6-month-old wild-type, NF-M ${ }^{\text {wt } / S \rightarrow A}, N F-M^{S \rightarrow A / S \rightarrow A}$, and $\mathrm{NF}-\mathrm{M}^{\text {tail } \Delta}$ homozygous mice were fractionated on $7.5 \%$ SDS polyacrylamide gels and stained with Coomassie blue $(\boldsymbol{A})$ or immunoblotted $(\boldsymbol{B})$ with antibodies that recognize NF-H in a phospho-independent manner (pAb-NF-HCOOH), NF-H in a phospho-dependent manner (RT-97 and SMI31), an epitope within the helical rod of NF-M (RM0-44), NF-L (NR-4), the neuron-specific $\beta$ III-tubulin (T8660), and $\beta$-tubulin (18D6).

corresponding levels of wild-type NF-M in normal nerves (Fig. $2 A) . N F-M^{S \rightarrow A}$ migrated with an increased mobility relative to wild-type NF-M, consistent with loss of KSP phosphorylation. Immunoblotting with an antibody specific to a rod domain epitope in murine NF-M (RMO44) (Fig. 2B) resulted in similar intensities of a pair of species of differing electrophoretic mobilities in both wild-type and $\mathrm{NF}-\mathrm{M}^{\mathrm{S} \rightarrow \mathrm{A}}$ mice, confirming that phospho-KSP-incompetent and wild-type NF-M accumulated to similar levels in sciatic nerve (Fig. 2 B). Moreover, similar levels of NF-L, NF-H, and the neuron-specific $\beta$ III-tubulin isoform (Lee et al., 1990) and $\beta$-tubulin were also observed in wild-type and NF-M ${ }^{\mathrm{S} \rightarrow \mathrm{A}}$ extracts (Fig. $2 \mathrm{~B}$ ), demonstrating that preventing phosphorylation of NF-M KSP repeats does not affect accumulation of the other major axonal cytoskeletal proteins in sciatic nerve. Phospho-epitopes recognized on NF-M by the monoclonal antibody SMI-31 were diminished in NF-M ${ }^{\mathrm{S} \rightarrow \mathrm{A}}$ heterozygous mice and completely absent in $\mathrm{NF}-\mathrm{M}^{\mathrm{S} \rightarrow \mathrm{A}}$ homozygous mice (Fig. 2B). On the other hand, phospho-epitopes on NF-H (and recognized by SMI-31 or RT97 antibodies) were unaffected. Prolonged exposure of immunoblots to detect phospho-epitopes indicated a modest elevation in phosphorylation of NF-H (supplemental Fig. 1, available at www.jneurosci.org as supplemental material). However, the level of this potentially compensatory phosphorylation of NF-H was similar in both NF-M ${ }^{\text {tail }}$ and NF$\mathrm{M}^{\mathrm{S} \rightarrow \mathrm{A}}$ mice (supplemental Fig. 1, available at www.jneurosci.org as supplemental material), the former of which almost completely fail radial axonal growth (Garcia et al., 2003).

\section{Phosphorylation of NF-M KSP repeats is not required for myelin-dependent radial axonal growth}

In a previous study, we demonstrated that loss of the entire NF-M tail domain, including all identified phosphorylation sites, resulted in axons with impaired radial axonal growth (Garcia et al., 2003). To determine whether myelin-dependent phosphorylation of KSP repeats within this tail is essential for radial axonal growth, the size of all axons within the fifth lumbar ventral root was determined at 2 and 6 months (Fig. 3A). Cross-sectional areas were measured for all axons at both time points, and corresponding diameters were calculated (Fig. 3C,D). Accumulation of wild-type and NF-M ${ }^{\mathrm{S} \rightarrow \mathrm{A}}$ protein yielded bimodal distributions of motor axons (Fig. 3C,D). There was a small, but statistically significant, difference in the distribution of axonal diameters at 2 months of age. Surprisingly, however, unlike the prevention of radial growth observed in NF-M ${ }^{\text {tail } \Delta}$ mice (Garcia et al., 2003), by 6 months of age axonal diameter in NF-M ${ }^{\mathrm{S} \rightarrow \mathrm{A}}$ mice was essentially indistinguishable from that of normal mice (Fig. $3 C, D$ ). The total number of motor axons was also unchanged in mature NF-M ${ }^{\mathrm{S} \rightarrow \mathrm{A}}$ nerves compared with normal nerves, although normal developmental paring of motor axon number between 2 and 6 months of age seen in wild-type normal mice was apparently accelerated in the NF-M $\mathrm{M}^{\mathrm{S} \rightarrow \mathrm{A}}$ mice (Fig. $3 B$ ). Together with previous findings from NF-M ${ }^{\text {tail } \Delta}$ mice (Garcia et al., 2003), these data demonstrate that myelin-dependent radial axonal growth does not require myelin-dependent phosphorylation of NF-M KSP repeats.

\section{Axoplasmic organization is slightly altered in mice expressing KSP phospho-incompetent NF-M}

To determine whether NF-M KSP phosphorylation is essential for establishing normal neurofilament spacing and three dimensional axoplasmic organization, cross sections from fifth lumbar motor axons were compared with wild-type (Fig. 4A) and NF$\mathrm{M}^{\mathrm{S} \rightarrow \mathrm{A} / \mathrm{S} \rightarrow \mathrm{A}}$ mice using electron microscopy (Fig. $4 B$ ). Quantification of the spacing between adjacent neurofilaments (nearestneighbor distance) revealed that while at 2 months of age the filament-to-filament spacing within NF-M ${ }^{\mathrm{S} \rightarrow \mathrm{A} / \mathrm{S} \rightarrow \mathrm{A}}$ axons was reduced compared with wild-type controls (Fig. 4C), by 6 months, the distribution of spacings was essentially indistinguishable between the two genotypes (Fig. 4D). [The absolute magnitudes of our current measurements differ from our previously reported values (Garcia et al., 2003); we believe that this likely reflects systematic differences in tissue preparation before analysis, and we emphasize that all samples reported here were processed in parallel.]

Next, neurofilament clustering was measured by identifying the positions of all neurofilaments in individual axons and calculating the ratio of average filament spacing to nearest-neighbor distance. Higher ratios imply less uniformly distributed neurofilaments. Neurofilaments within an axon were redistributed into hexagonal arrays over a cross-sectional area equal to the crosssectional area of the axon. Average neurofilament spacing was then defined to be the edge length of each hexagon. A perfectly ordered array of neurofilaments would, therefore, yield a value of 1.0. Not surprisingly, the neurofilament array in wild-type mice diverged from a perfectly ordered one (Fig. $4 E$ ) at both 2 and 6 months. However, preventing KSP phosphorylation did not affect the degree of divergence from a perfectly ordered array (Fig. $4 E$ ).

Neurofilament tail domains influence the organization of the 

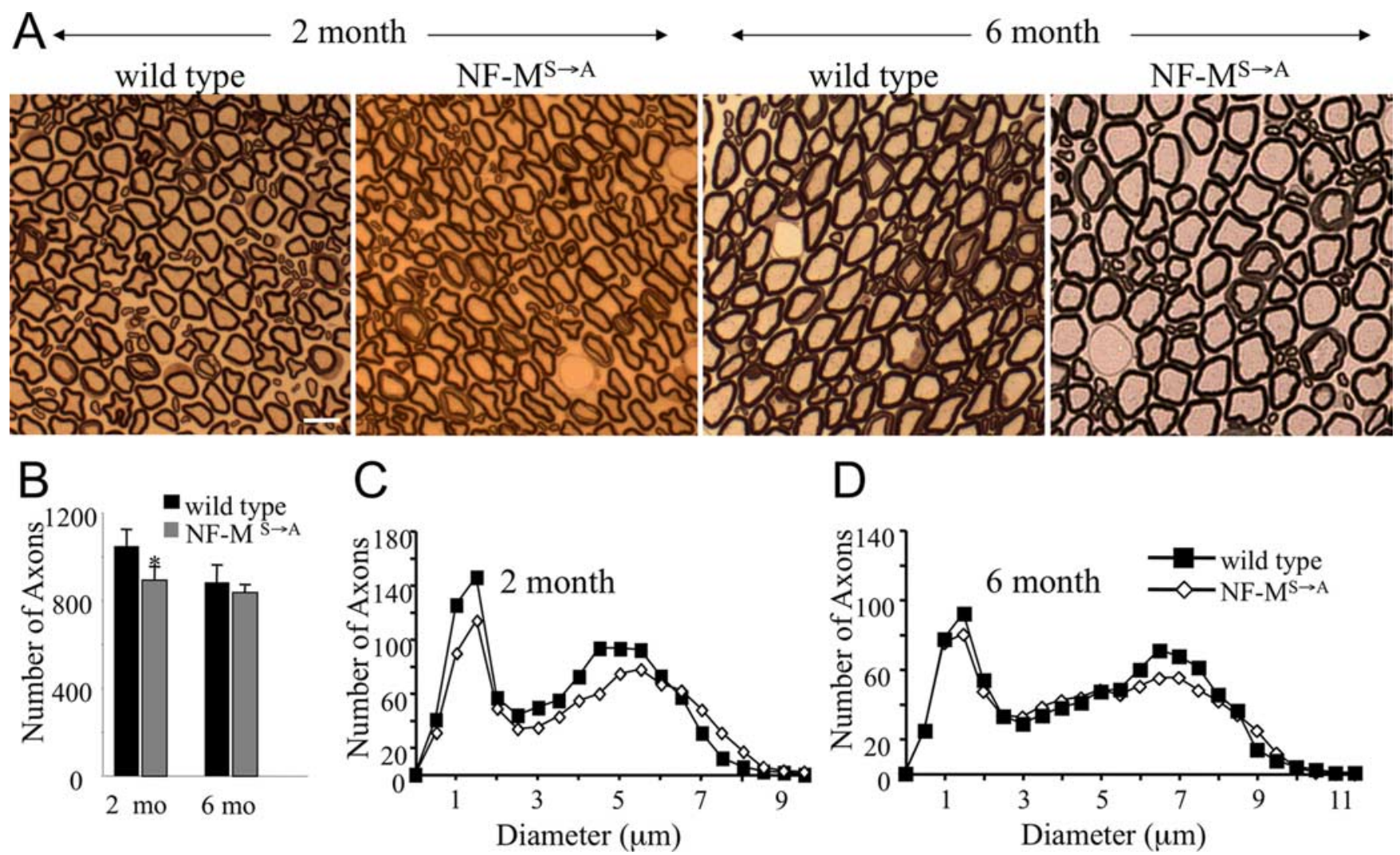

Figure 3. Absence of NF-M phosphorylation does not affect radial growth or survival of motor axons. $A$, Cross sections of the fifth lumbar motor (ventral) root from wild-type and NF-M $\rightarrow A$ homozygous mice at 2 months (left) or 6 months (right) of age. Scale bar, $10 \mu \mathrm{m}$. B, Number of axons in the fifth lumbar motor roots of 2- or 6 -month-old wild-type and NF-M ${ }^{S} \rightarrow$ A homozygous mice. Counts are average from four to five animals for each genotype. Means, for total axon counts, were analyzed by unpaired Student's $t$ test. ${ }^{*} p<0.03$. $\boldsymbol{C}, \boldsymbol{D}$, Distributions of axonal diameters in motor axons in 2-month-old $(\boldsymbol{C})$ or 6-month-old $(\boldsymbol{D})$ wild-type and NF-M ${ }^{\mathrm{S} \rightarrow A}$ homozygous mice. Each point represents the averaged distribution of axon diameters from the entire roots of five mice for each genotype and age group. Axonal populations were analyzed for overall statistical differences using a Mann-Whitney $U$ test. There was a small, but statistically significant, difference between diameter distributions of wild-type versus NF-M ${ }^{S \rightarrow A}$ mice at 2 months $(p<0.05)$. Error bars indicate SEM.

axonal cytoskeleton (Garcia et al., 2003). Nevertheless, longitudinal views of axoplasm by quick-freeze, deep-etch microscopy revealed that axoplasm is structured similarly in wild-type (Fig.

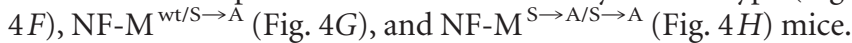
Axoplasm from all three genotypes has similar neurofilament tail-derived cross-bridges (at comparable frequencies) extending to or from the surface of neurofilaments (Fig. $4 F-H$, arrowheads). Neurofilament tail-derived cross-bridges were identified as single, thin structures projecting from the core of the filament as previously observed in Sf9 cells coexpressing NF-L and NF-M (Nakagawa et al., 1995) or NF-L and NF-H (Chen et al., 2000). Additionally, more complex plectin-like linkers (Fig. $4 \mathrm{~F}-\mathrm{H}$, arrows) [as previously observed in cytoskeletal extracts of rat embryonic fibroblast cells (Svitkina et al., 1996)] were present at comparable frequencies in all three genotypes and were visible as "Y" or "V" structures that projected from the core of individual filaments. Despite an apparent decrease in filament-to-filament spacing (Fig. $4 C, D, H$ ), overall, axoplasm in NF-M $\mathrm{M}^{\mathrm{S} \rightarrow \mathrm{A} / \mathrm{S} \rightarrow \mathrm{A}}$ mice was similar to that in either wild-type or heterozygous mice.

Expressing KSP phospho-incompetent NF-M did not alter the accumulation of cytoskeletal components in motor axons Microtubule content, in principle, could be influenced by the presence of the NF-M tail domain (Garcia et al., 2003; Rao et al., 2003). Therefore, we quantified the accumulation levels of both neurofilaments (Fig. 5A) and microtubules (Fig. 5B) in motor neurons. As expected from the constant amount of each of the three neurofilament subunits observed in immunoblots, the total number of neurofilaments per axonal segment was not different between wild-type versus NF-M ${ }^{\mathrm{S} \rightarrow \mathrm{A} / \mathrm{S} \rightarrow \mathrm{A}}$ motor axons at either 2 or 6 months of age (Fig. 5A). Additionally, prevention of NF-M KSP phosphorylation had no effect on axonal microtubule content at both time points examined (Fig. $5 B$ ), albeit there was a nonsignificant trend toward a higher microtubule number/neurofilament number in the axons of NF-M $\mathrm{M}^{\mathrm{S} \rightarrow \mathrm{A}}$ mice (Fig. $5 C$ ).

\section{Motor nerve conduction velocity is not altered in KSP} phospho-incompetent NF-M-expressing mice

Motor axon conduction velocities were measured in the sciatic nerves of 6-month-old mice (five mice per genotype) from wildtype and $\mathrm{NF}-\mathrm{M}^{\mathrm{S} \rightarrow \mathrm{A}}$ mice. Consistent with caliber as a central determinant of conduction velocity (Erlanger and Gasser, 1930; Blair and Erlanger, 1933; Garcia et al., 2003) and our earlier determination that axonal diameter is not altered after replacement of NF-M with the phospho-KSP-incompetent variant, the velocity of action potential propagation was also unaffected in NF$\mathrm{M}^{\mathrm{S} \rightarrow \mathrm{A}}$ mice relative to wild-type mice. NF-M ${ }^{\mathrm{S} \rightarrow \mathrm{A}}$ mice conduct at $56.6 \mathrm{~m} / \mathrm{s}( \pm 2.9 \mathrm{~m} / \mathrm{s})$ whereas wild-type mice conduct at 61.5 $\mathrm{m} / \mathrm{s}( \pm 3.7 \mathrm{~m} / \mathrm{s})$.

KSP-phospho-incompetent NF-M does not alter locomotor activity or recovery rates after nerve injury

As a further measure of motor neuron performance in $N F-M^{S \rightarrow A}$ mice relative to wild-type mice, mice were provided running 

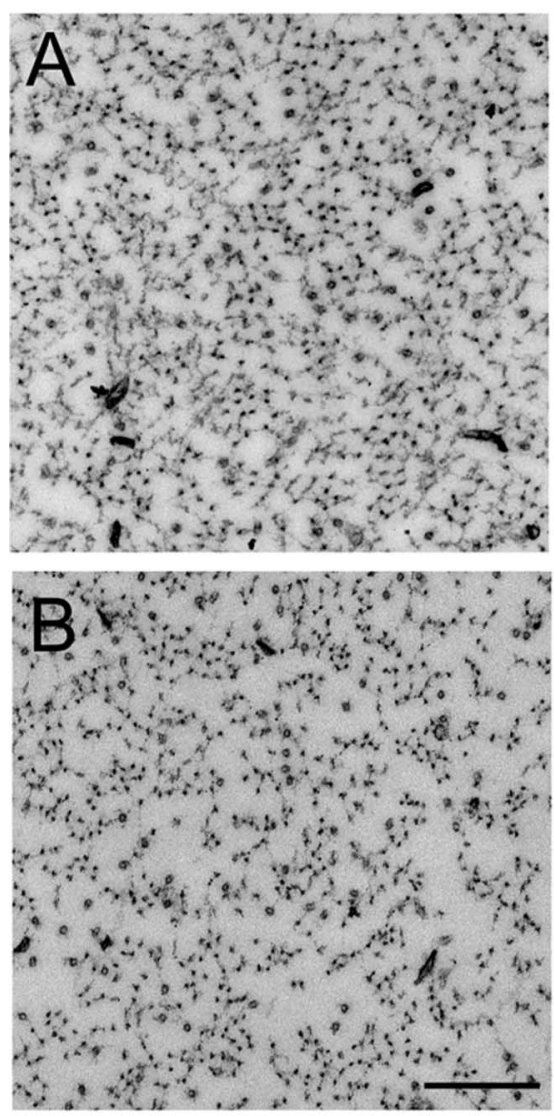
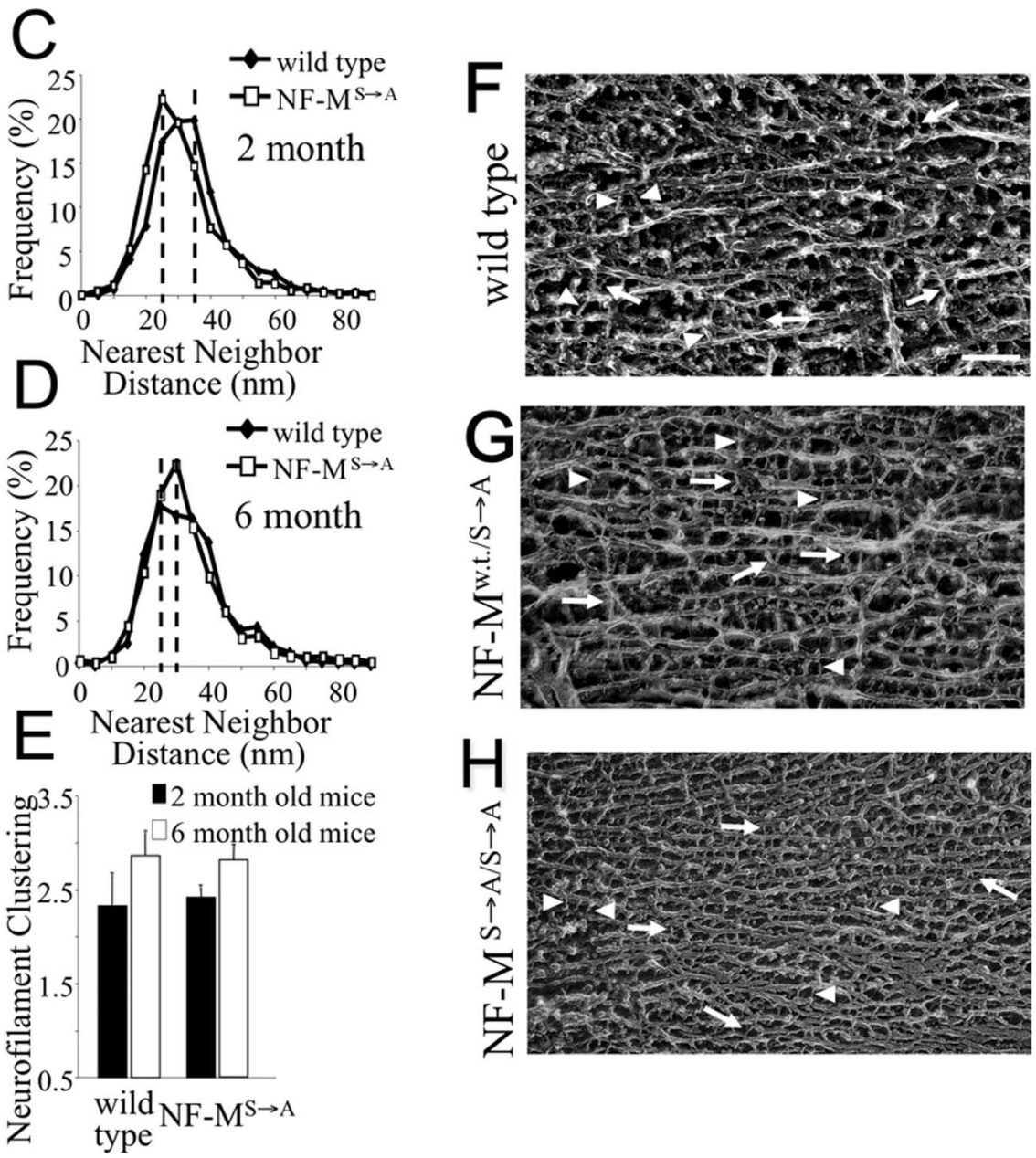

Figure 4. Structure of axoplasm is slightly altered due to the expression of KSP phospho-incompetent NF-M. $A, B$, Transmission electron micrographs of 2-month-old motor axons derived from the fifth lumbar spinal cord segment of wild-type $(\boldsymbol{A})$ and NF-M ${ }^{S \rightarrow A}(\boldsymbol{B})$ homozygous mice. Scale bar, $400 \mathrm{~nm}$. C, D, Distribution of nearest-neighbor distances from motor axons of 2-month-old $(\boldsymbol{C})$ and 6-month-old $(\boldsymbol{D})$ wild-type and NF-M ${ }^{S \rightarrow A}$ homozygous mice. $\boldsymbol{E}$, Neurofilament clustering, defined as the ratio of average filament spacing to nearest-neighbor spacing, was unaltered in wild-type versus NF-M ${ }^{S \rightarrow A}$ mice at both 2 and 6 months, indicating that axoplasmic organization is similar in both mice. $\boldsymbol{F}-\boldsymbol{H}$, Quick-freeze, deep-etch micrographs of sciatic nerves from wild-type $(\boldsymbol{F}), \mathrm{NF}-\mathrm{M}^{+/ S \rightarrow A}(\boldsymbol{G})$, and NF-M $\mathrm{M}^{S \rightarrow A} /^{S \rightarrow A}(\boldsymbol{H})$ mice. Scale bar, $400 \mathrm{~nm}$. Arrowheads, Single, thin cross-linkers projecting from the core of neurofilaments. Arrows, Plectin-like linkers identified as more complex cross-linking structures projecting from the core of individual filaments. Many form Y or V structures. Error bars indicate SEM.

wheels. Measurement of their total distances traveled per night revealed that voluntary running distances were not statistically different (although there was trend for the NF- $\mathrm{M}^{\mathrm{S} \rightarrow \mathrm{A}}$ mice to cover greater distances than wild-type mice). Wild-type mice voluntarily ran $8.6 \mathrm{~km} / \mathrm{d}( \pm 2.4 \mathrm{~km} / \mathrm{d})$, and NF-M ${ }^{\mathrm{S} \rightarrow \mathrm{A}}$ mice voluntarily $\mathrm{ran} 10.3 \mathrm{~km} / \mathrm{d}( \pm 2.6 \mathrm{~km} / \mathrm{d})$. Finally, the investment of neurofilaments into axons is suppressed during recovery from nerve crush, returning to normal levels only after recovery is complete (Hoffman et al., 1987). To determine whether expressing KSPphospho-incompetent NF-M affects axonal recovery after injury, sciatic nerves were crushed, at the level of the obturator tendon, and the speed of recovery of toe spread in the ipsilateral limb in wild-type and NF-M ${ }^{\mathrm{S} \rightarrow \mathrm{A}}$ mice was assessed daily for 3 weeks. Recovery profiles from both mice were indistinguishable (Fig. 6).

\section{Discussion}

Within myelinated axons of normal animals, the internodal diameter is increased approximately sevenfold relative to that of unmyelinated axonal segments (de Waegh et al., 1992; Hsieh et al., 1994). For the last 15 years, the proposed underlying mechanism(s) for this internodal radial axonal growth has focused on myelin-dependent phosphorylation of neurofilament heavy
(NF-H) and medium (NF-M) tail domains (de Waegh et al., 1992), which extend from the filament surface to form crossbridges between adjacent neurofilaments and microtubules (Hisanaga and Hirokawa, 1988). Indeed, within myelinated internodes neurofilaments are some of the most heavily phosphorylated axonal proteins (Julien and Mushynski, 1982), with the majority of the identified phosphorylation occurring on the KSP, KXSP, and KXXSP repeat motifs (Glicksman et al., 1987; Levy et al., 1987; Myers et al., 1987; Julien et al., 1988). The correspondingly large negative charge on both NF-M and NF-H tail domains has been argued to mediate their lateral extension from the core of the neurofilament (Glicksman et al., 1987; Myers et al., 1987), with the cross-bridges forcing increased neurofilament spacing (Nakagawa et al., 1995).

Among the most persuasive evidence for this model of radial axonal growth was demonstration that the formation of compact myelin and the axonal accumulation of neurofilaments were both necessary for radial axonal growth. Grafting myelin-deficient Schwann cells derived from the Trembler mouse onto wild-type axons resulted in less phosphorylated, more densely packed neurofilaments and axonal segments that failed to grow radially 


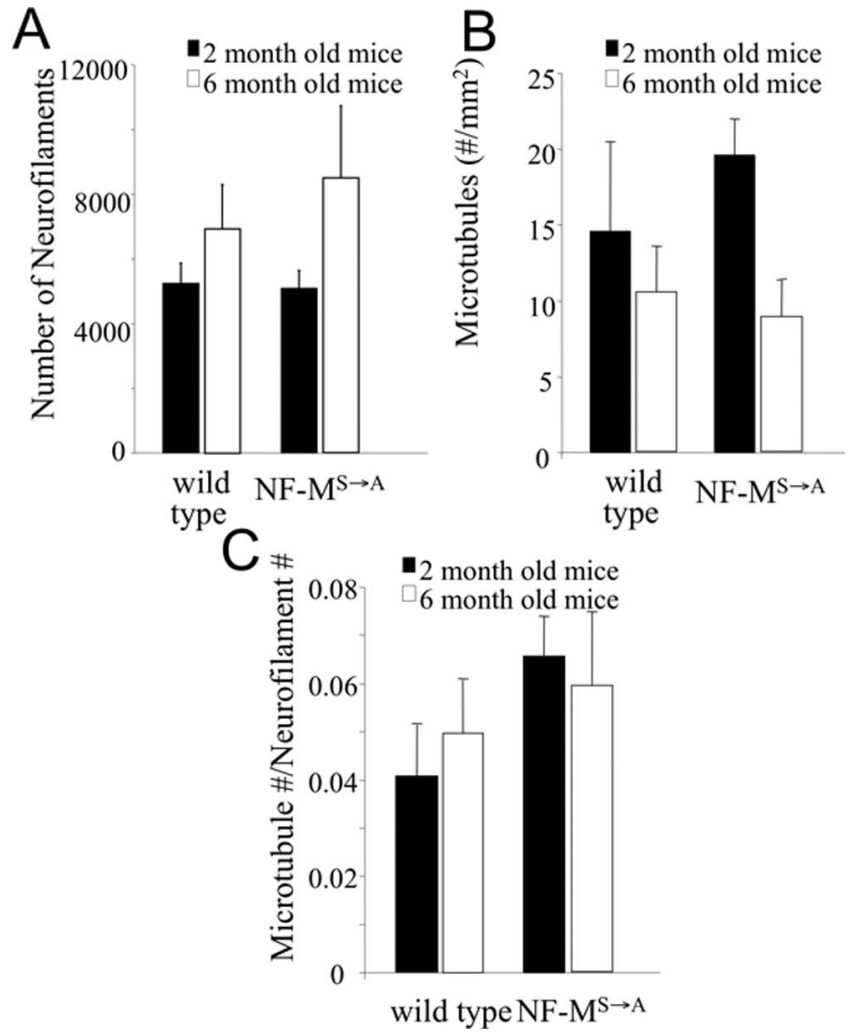

Figure 5. Accumulation of cytoskeletal components is unaltered in motor neurons in mice expressing phospho-incompetent NF-M. $A$, The number of neurofilaments per axonal segment in both wild-type and NF-M ${ }^{S \rightarrow A}$ homozygous mice at both 2 and 6 months. B, Microtubule content was reflective of overall axoplasmic organization. A trend toward accumulating more microtubules occurred in 2-month-old NF-M ${ }^{S \rightarrow A}$ mice but did not reach statistical significance. This trend was not evident at 6 months, indicating no difference in axoplasmic organization. $\boldsymbol{C}$ The ratio of microtubule number to neurofilament number was unchanged in wild-type versus $\mathrm{NF}-\mathrm{M}^{\mathrm{S} \rightarrow \mathrm{A}}$ mice. All data were analyzed for overall statistical analysis using ANOVA. Error bars indicate SEM.

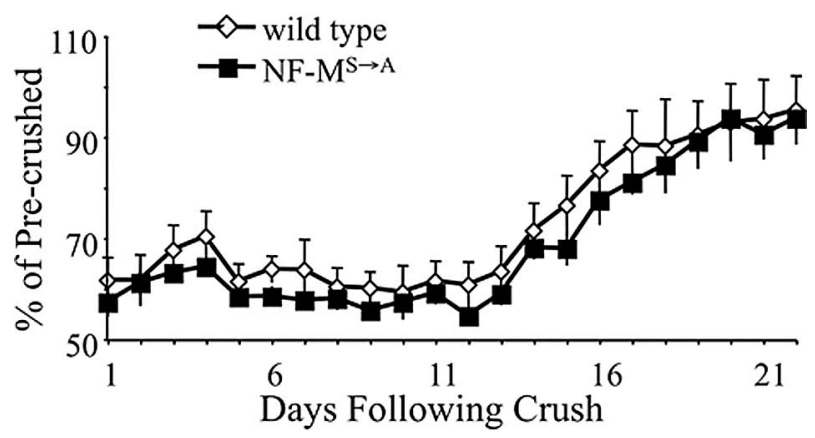

Figure 6. Expression of phospho-incompetent NF-M does not affect recovery rates from sciatic nerve injury. Functional recovery, by measuring the distance between the first and fifth digit, was measured in sciatic nerves that had been crushed at the level of the obturator tendon from wild-type and NF-M ${ }^{S \rightarrow A}$ mice. Values were plotted as a percentage of motor function before crush injury. A minimum of five mice per genotype was assayed, and measurements were performed in triplicate for each animal per day for $21 \mathrm{~d}$ after crush injury. Error bars indicate SEM.

within the graft (de Waegh et al., 1992). Moreover, loss of axonal neurofilament accumulation resulted in a phenotype similar to loss of compact myelin, as axons devoid of neurofilaments did not undergo radial axonal growth (Ohara et al., 1993; Zhu et al., 1997). Analysis of mice expressing C-terminally truncated neurofilaments supported the myelin-dependent phosphorylation hypothesis (Garcia et al., 2003). However, gene replacement to delete the heavily phosphorylated KSPs within tail domain of NF-H did not affect specification of mature axonal diameter (Rao et al., 2002). In contrast, similar gene replacement in mice to truncate the NF-M tail (NF-M ${ }^{\text {tail } \Delta}$ mice) resulted in more closely packed neurofilaments and axons with impaired radial growth (Garcia et al., 2003) and led to the current model in which phosphorylation of KSP sites in NF-M is an essential feature of radial axonal growth.

The present analysis of mice expressing full-length KSP phospho-incompetent NF-M (NF-M ${ }^{\mathrm{S} \rightarrow \mathrm{A}}$ mice) challenges the long standing model of neurofilament KSP phosphorylation as the determinant of radial growth. Although loss of all NF-H tail phosphorylation including the $51 \mathrm{KSP}$ repeats does not affect final axonal caliber (Rao et al., 2002), loss of the NF-M tail and its KSP motifs does. We have now found very surprisingly that phosphorylation of serine residues of the KSP repeats (including KSP, KXSP, KXXSP, and the KSD variant) within the NF-M tail domain is not a determinant of radial axonal growth. Rather, the distribution of axonal diameters in NF- $\mathrm{M}^{\mathrm{S} \rightarrow \mathrm{A}}$ mice is indistinguishable with those of age matched, wild-type mice. This cannot simply be explained by compensatory phosphorylation of NF-H, as NF-M ${ }^{\text {tails }}$ and NF-M ${ }^{\mathrm{S} \rightarrow \mathrm{A}}$ mice showed a similarly modest increase in NF-H phosphorylation, and yet this was not sufficient to compensate for the loss of the entire NF-M tail domain (Garcia et al., 2003).

The retention of radial axonal growth despite reduced neurofilament-to-neurofilament spacing that we report here in $\mathrm{NF}-\mathrm{M}^{\mathrm{S} \rightarrow \mathrm{A}}$ mice relative to the spacing in wild-type nerves supports our previous findings that axonal diameter does not correlate with the distance between neurofilaments. In mice simultaneously expressing truncated NF-M and NF-H (NF- $(\mathrm{M} / \mathrm{H})^{\text {tail } \Delta}$ mice), axonal diameters are indistinguishable at 6 months relative to NF-M ${ }^{\text {tails }}$ mice, but neurofilament-to-neurofilament spacing is reduced in NF- $(\mathrm{M} / \mathrm{H})^{\text {tails }}$ mice relative to NF-M ${ }^{\text {tail } \Delta}$ mice (Garcia et al., 2003).

When combined with the previous findings that radial axonal growth is not compromised in mice without NF-H KSP phosphorylation (Rao et al., 2002; Garcia et al., 2003), the present data indicate that myelin-dependent phosphorylation of neurofilament KSP repeat domains (on either NF-M or NF-H) cannot play a crucial role in determining axonal caliber. These new insights represent what may be the first phase of a paradigm shift in understanding neurofilament biology and its relationship to determining axonal caliber. Neurofilament KSP phosphorylation has widely been considered a major determinant of radial axonal growth, potentially through influence on axonal cytoarchitecture (Nixon et al., 1994; Sánchez et al., 2000), on axonal transport (Lewis and Nixon, 1988), or on both. Our evidence documents that neurofilament KSP phosphorylation does not affect axonal cytoarchitecture. Other recent evidence also supports the view that KSP phosphorylation does not influence the rate of neurofilament transport (Rao et al., 2002, 2003; Yuan et al., 2006). Rethinking the role of neurofilament KSP phosphorylation is, therefore, required.

More broadly, when taken together with our previous demonstration of a requirement for the NF-M tail domain in establishing radial axonal growth (Garcia et al., 2003), it is now clear that the basic mechanism for how neurofilaments specify radial growth remains unanswered. One hypothesis now emerging from the current evidence is that neurofilaments are phosphorylation targets for the myelin-derived outside-in signal as has long been argued, especially by the persuasive evidence of de Waegh et 
al. (1992), but at sites previously obscured by the nearstoichiometric phosphorylation of multiple KSP sites. Phosphorylation of non-KSP sites is consistent with the observation that despite prevention of all KSP phosphorylation NF-M does not migrate on SDS-PAGE gels at its predicted molecular weight of 96 $\mathrm{kDa}$, based on amino acid sequence (Fig. 2). KSP-independent modification of NF-M is now testable by further analysis of our mice deleted in neurofilament KSP sites, but which undergo normal radial growth.

\section{References}

Blair EA, Erlanger J (1933) A comparison of the characteristics of axons through their individual electrical responses. Am J Physiol 106:524-564.

Calcutt NA, Tomlinson DR, Biswas S (1990) Coexistence of nerve conduction deficit with increased $\mathrm{Na}(+)-\mathrm{K}(+)$-ATPase activity in galactose-fed mice. Implications for polyol pathway and diabetic neuropathy. Diabetes 39:663-666.

Chen J, Nakata T, Zhang Z, Hirokawa N (2000) The C-terminal tail domain of neurofilament protein- $\mathrm{H}$ (NF-H) forms the crossbridges and regulates neurofilament bundle formation. J Cell Sci 113:3861-3869.

Conway JH, Sloane NJA (1999) Sphere packings, lattices, and groups. New York: Springer.

de Waegh SM, Lee VM, Brady ST (1992) Local modulation of neurofilament phosphorylation, axonal caliber, and slow axonal transport by myelinating Schwann cells. Cell 68:451-463.

Duncan D (1934) A relationship between axone diameter and myelination determined by measurement of myelinated spinal root fibers. J Comp Neurol 60:437-471.

Erlanger J, Gasser HS (1930) The action potential in fibers of slow conduction in spinal roots and somatic nerves. Am J Physiol 92:43-82.

Garcia ML, Lobsiger CS, Shah SB, Deerinck TJ, Crum J, Young D, Ward CM, Crawford TO, Gotow T, Uchiyama Y, Ellisman MH, Calcutt NA, Cleveland DW (2003) NF-M is an essential target for the myelin-directed "outside-in" signaling cascade that mediates radial axonal growth. J Cell Biol 163:1011-1020.

Glicksman MA, Soppet D, Willard MB (1987) Posttranslational modification of neurofilament polypeptides in rabbit retina. J Neurobiol 18:167-196.

Gotow T, Leterrier JF, Ohsawa Y, Watanabe T, Isahara K, Shibata R, Ikenaka K, Uchiyama Y (1999) Abnormal expression of neurofilament proteins in dysmyelinating axons located in the central nervous system of jimpy mutant mice. Eur J Neurosci 11:3893-3903.

Hisanaga S, Hirokawa N (1988) Structure of the peripheral domains of neurofilaments revealed by low angle rotary shadowing. J Mol Biol 202:297-305.

Hoffman PN, Cleveland DW, Griffin JW, Landes PW, Cowan NJ, Price DL (1987) Neurofilament gene expression: a major determinant of axonal caliber. Proc Natl Acad Sci U S A 84:3472-3476.

Hsieh ST, Kidd GJ, Crawford TO, Xu Z, Lin WM, Trapp BD, Cleveland DW, Griffin JW (1994) Regional modulation of neurofilament organization by myelination in normal axons. J Neurosci 14:6392-6401.

Jacomy H, Zhu Q, Couillard-Després S, Beaulieu JM, Julien JP (1999) Disruption of type IV intermediate filament network in mice lacking the neurofilament medium and heavy subunits. J Neurochem 73:972-984.

Joyner AL (1994) Gene targeting. A practical approach. New York: IRL.

Julien JP, Mushynski WE (1982) Multiple phosphorylation sites in mammalian neurofilament polypeptides. J Biol Chem 257:10467-10470.

Julien JP, Côté F, Beaudet L, Sidky M, Flavell D, Grosveld F, Mushynski W (1988) Sequence and structure of the mouse gene coding for the largest neurofilament subunit. Gene 68:307-314.

Kriz J, Zhu Q, Julien JP, Padjen AL (2000) Electrophysiological properties of axons in mice lacking neurofilament subunit genes: disparity between conduction velocity and axon diameter in absence of NF-H. Brain Res 885:32-44.

Lee MK, Tuttle JB, Rebhun LI, Cleveland DW, Frankfurter A (1990) The expression and posttranslational modification of a neuron-specific betatubulin isotype during chick embryogenesis. Cell Motil Cytoskeleton $17: 118-132$.
Lee MK, Xu Z, Wong PC, Cleveland DW (1993) Neurofilaments are obligate heteropolymers in vivo. J Cell Biol 122:1337-1350.

Levy E, Liem RK, D’Eustachio P, Cowan NJ (1987) Structure and evolutionary origin of the gene encoding mouse NF-M, the middle-molecular-mass neurofilament protein. Eur J Biochem 166:71-77.

Lewis SE, Nixon RA (1988) Multiple phosphorylated variants of the high molecular mass subunit of neurofilaments in axons of retinal cell neurons: characterization and evidence for their differential association with stationary and moving neurofilaments. J Cell Biol 107:2689-2701.

Lopata MA, Cleveland DW (1987) In vivo microtubules are copolymers of available beta-tubulin isotypes: localization of each of six vertebrate betatubulin isotypes using polyclonal antibodies elicited by synthetic peptide antigens. J Cell Biol 105:1707-1720.

Myers MW, Lazzarini RA, Lee VM, Schlaepfer WW, Nelson DL (1987) The human mid-size neurofilament subunit: a repeated protein sequence and the relationship of its gene to the intermediate filament gene family. EMBO J 6:1617-1626.

Nakagawa T, Chen J, Zhang Z, Kanai Y, Hirokawa N (1995) Two distinct functions of the carboxyl-terminal tail domain of NF-M upon neurofilament assembly: cross-bridge formation and longitudinal elongation of filaments. J Cell Biol 129:411-429.

Nixon RA, Paskevich PA, Sihag RK, Thayer CY (1994) Phosphorylation on carboxyl terminus domains of neurofilament proteins in retinal ganglion cell neurons in vivo: influences on regional neurofilament accumulation, interneurofilament spacing, and axon caliber. J Cell Biol 126:1031-1046.

Ohara O, Gahara Y, Miyake T, Teraoka H, Kitamura T (1993) Neurofilament deficiency in quail caused by nonsense mutation in neurofilament- $\mathrm{L}$ gene. J Cell Biol 121:387-395.

Rao MV, Garcia ML, Miyazaki Y, Gotow T, Yuan A, Mattina S, Ward CM, Calcutt NA, Uchiyama Y, Nixon RA, Cleveland DW (2002) Gene replacement in mice reveals that the heavily phosphorylated tail of neurofilament heavy subunit does not affect axonal caliber or the transit of cargoes in slow axonal transport. J Cell Biol 158:681-693.

Rao MV, Campbell J, Yuan A, Kumar A, Gotow T, Uchiyama Y, Nixon RA (2003) The neurofilament middle molecular mass subunit carboxylterminal tail domains is essential for the radial growth and cytoskeletal architecture of axons but not for regulating neurofilament transport rate. J Cell Biol 163:1021-1031.

Rushton WA (1951) A theory of the effects of fibre size in medullated nerve. J Physiol 115:101-122.

Sakaguchi T, Okada M, Kitamura T, Kawasaki K (1993) Reduced diameter and conduction velocity of myelinated fibers in the sciatic nerve of a neurofilament-deficient mutant quail. Neurosci Lett 153:65-68.

Sánchez I, Hassinger L, Sihag RK, Cleveland DW, Mohan P, Nixon RA (2000) Local control of neurofilament accumulation during radial growth of myelinating axons in vivo. Selective role of site-specific phosphorylation. J Cell Biol 151:1013-1024.

Stevens B, Fields RD (2000) Response of Schwann cells to action potentials in development. Science 287:2267-2271.

Svitkina TM, Verkhovsky AB, Borisy GG (1996) Plectin sidearms mediate interaction of intermediate filaments with microtubules and other components of the cytoskeleton. J Cell Biol 135:991-1007.

Tu PH, Elder G, Lazzarini RA, Nelson D, Trojanowski JQ, Lee VM (1995) Overexpression of the human NFM subunit in transgenic mice modifies the level of endogenous NFL and the phosphorylation state of NFH subunits. J Cell Biol 129:1629-1640.

Xu Z, Cork LC, Griffin JW, Cleveland DW (1993) Increased expression of neurofilament subunit NF-L produces morphological alterations that resemble the pathology of human motor neuron disease. Cell 73:23-33.

Xu ZS, Liu WS, Willard MB (1992) Identification of six phosphorylation sites in the $\mathrm{COOH}$-terminal tail region of the rat neurofilament protein M. J Biol Chem 267:4467-4471.

Yin X, Crawford TO, Griffin JW, Tu P, Lee VM, Li C, Roder J, Trapp BD (1998) Myelin-associated glycoprotein is a myelin signal that modulates the caliber of myelinated axons. J Neurosci 18:1953-1962.

Yuan A, Nixon RA, Rao MV (2006) Deleting the phosphorylated tail domain of the neurofilament heavy subunit does not alter neurofilament transport rate in vivo. Neurosci Lett 393:264-268.

Zhu Q, Couillard-Després S, Julien JP (1997) Delayed maturation of regenerating myelinated axons in mice lacking neurofilaments. Experimental Neurology 148:299-316. 